MIFP-05-03

hep-th/0501213

January 2005

\title{
AdS pp-waves
}

\author{
Johannes Kerimo $^{1}$ \\ George P. $\&$ Cynthia W. Mitchell Institute for Fundamental Physics, \\ Texas A $8 M$ University, College Station, TX 77843-4242, USA
}

\begin{abstract}
$\underline{\text { ABSTRACT }}$
We obtain the pp-waves of $D=5$ and $D=4$ gauged supergravities supported by $U(1)^{3}$ and $U(1)^{4}$ gauge field strengths respectively. We show that generically these solutions preserve $1 / 4$ of the supersymmetry, but supernumerary supersymmetry can arise for appropriately constrained harmonic functions associated with the pp-waves. In particular it implies that the solutions are independent of the light-cone coordinate $x^{+}$. We also obtain the pp-waves in the Freedman-Schwarz model.
\end{abstract}

\footnotetext{
${ }^{1}$ Research supported in part by DOE grant DE-FG03-95ER40917.
} 


\section{Introduction}

The subject of pp-waves and their applications have been studied extensively. In particular, superstring theory is exactly solvable [1] on the backgrounds of the maximal supersymmetric pp-waves of type IIB [2] and M-theory [3]. This provides a rare example where the AdS/CFT duality [4, 5, 6] can be tested [7] beyond the supergravity approximation.

When the integration constants associated with these solutions are left arbitrary the pp-waves generically preserve half of the supersymmetry. Additional supersymmetry (supernumerary supersymmetry) beyond the $\frac{1}{2}$ can arise when the harmonic function is constrained appropriately. For a discussion of pp-waves and their supersymmetry in M-theory and type IIB see [8, 9, 10, 11, 12, 13].

PP-waves on AdS background have also been studied. The purely gravitational AdS pp-wave is given by

$$
d s^{2}=e^{2 g \rho}\left(-4 d x^{+} d x^{-}+H\left(d x^{+}\right)^{2}+d z_{a}^{2}\right)+d \rho^{2},
$$

where the cosmological constant is related to the gauge coupling constant $g$ as $\Lambda=-g^{2}$, and $H=H\left(x^{+}, \rho, z_{a}\right)$ is a harmonic function on the space of $z_{a}$ and $\rho$. The integration constants of $H$ are therefore allowed to have an arbitrary dependence on $x^{+}$. If $H=0$ the metric describes pure AdS spacetime. The pp-wave with the dependence $H(\rho)$ was constructed in four dimensions by Kaigorodov [14] and its higher dimensional counterparts were obtained in [15]. Generalisations of the Kaigorodov metric to inhomogeneous solutions were obtained in 16, 17, 18.

The superimposing of a pp-wave on AdS spacetime can be viewed as performing an infinite boost on the boundary conformal field theory [15, 19]. These solutions generically preserve $\frac{1}{4}$ of the maximum supersymmetry allowed by the AdS spacetime [15, 19]. The purely gravitational AdS pp-waves have in fact been shown to admit supernumerary supersymmetries [20] for appropriately constrained $H$.

AdS pp-waves can also be supported by a field strength. Their supersymmetry has been studied in [21, 22, 23, 20]. See also [24]. In the case of charged pp-waves of minimum gauged supergravities in $D=4$ and $D=5$, it was shown [23, 20] that supernumerary supersymmetry can arise again for appropriately constrained harmonic functions. For ppwaves with $\frac{1}{2}$ supersymmetry in $D=3$ see [25].

In this paper we shall investigate pp-waves on AdS background further by studying the pp-waves of $D=5$ and $D=4$ gauged supergravities supported respectively by $U(1)^{3}$ and $U(1)^{4}$ gauge fields. We present a detailed analysis of the supersymmetry of these 
solutions. In particular, we show that supernumerary supersymmetry can arise beyond the usual $\frac{1}{4}$. We also study the pp-waves of the Freedman-Schwarz model. The supersymmetry enhancement obtained in this paper forces the solutions to be independent of the light-cone coordinate $x^{+}$.

The paper is organised as follows. The pp-waves of gauged supergravities in five and four dimensions are studied in sections 2 and 3 respectively. We investigate the pp-waves of the Freedman-Schwarz model in section 4 . In sections 5 and 6 we study the supersymmetry of the solutions in six and seven dimensions, respectively.

\section{PP-waves in five dimensions}

Our first example treats $D=5$ gauged supergravity truncated to the $U(1)^{3}$ subgroup of $S O(6)$. The bosonic sector of this truncated theory is described by the Lagrangian [26]

$$
e^{-1} \mathcal{L}_{5}=R-\frac{1}{2}(\partial \vec{\varphi})^{2}+4 g^{2} \sum_{i} X_{i}^{-1}-\frac{1}{4} \sum_{i} X_{i}^{-2}\left(F_{(2)}^{i}\right)^{2}+\frac{1}{4} \epsilon^{M N P Q R} F_{M N}^{1} F_{P Q}^{2} A_{R}^{3},
$$

where $\vec{\varphi}=\left(\varphi_{1}, \varphi_{2}\right)$, and we write

$$
\begin{array}{ll}
X_{i}=e^{-\frac{1}{2} \vec{a}_{i} \cdot \vec{\varphi}}, & X_{1} X_{2} X_{3}=1, \\
\vec{a}_{1}=\left(\frac{2}{\sqrt{6}}, \sqrt{2}\right), \quad \vec{a}_{2}=\left(\frac{2}{\sqrt{6}},-\sqrt{2}\right), \quad \vec{a}_{3}=\left(-\frac{4}{\sqrt{6}}, 0\right),
\end{array}
$$

and the field strengths are defined as $F_{(2)}^{i}=d A_{(1)}^{i}$. The equations of motion are

$$
\begin{aligned}
R_{M N}= & \frac{1}{2} \partial_{M} \vec{\varphi} \cdot \partial_{N} \vec{\varphi}-\frac{4}{3} g^{2} g_{M N} \sum_{i} X_{i}^{-1} \\
& +\frac{1}{2} \sum_{i} X_{i}^{-2}\left(F_{M P}^{i} F_{N}^{i P}-\frac{1}{6}\left(F_{(2)}^{i}\right)^{2} g_{M N}\right), \\
\nabla_{M}\left(X_{i}^{-2} F_{i}^{M N}\right)= & \frac{1}{4} \epsilon^{N P Q R S} F_{P Q}^{j} F_{R S}^{k}, \quad i \neq j \neq k \neq i \\
\square \vec{\varphi}= & \frac{1}{4} \sum_{i} \vec{a}_{i} X_{i}^{-2}\left(F_{(2)}^{i}\right)^{2}-2 g^{2} \sum_{i} \vec{a}_{i} X_{i}^{-1} .
\end{aligned}
$$

The supersymmetry transformations for the fermions are given by

$$
\begin{aligned}
\delta \Psi_{M} & =\left[\nabla_{M}-\frac{\mathrm{i}}{2} g \sum_{i} A_{M}^{i}+\frac{1}{6} g \Gamma_{M} \sum_{i} X_{i}-\frac{\mathrm{i}}{48}\left(\Gamma_{M} \Gamma^{A B}-3 \Gamma^{A B} \Gamma_{M}\right) \sum_{i} X_{i}^{-1} F_{A B}^{i}\right] \epsilon, \\
\delta \vec{\lambda} & =\left[-\frac{\mathrm{i}}{4} \Gamma^{M} \partial_{M} \vec{\varphi}+\frac{1}{16} \Gamma^{A B} \sum_{i} \vec{a}_{i} X_{i}^{-1} F_{A B}^{i}-\frac{\mathrm{i}}{4} g \sum_{i} \vec{a}_{i} X_{i}\right] \epsilon .
\end{aligned}
$$

\subsection{The solution}

We use the following pp-wave metric ansatz

$$
d s_{D}=e^{2 A}\left(-4 d x^{+} d x^{-}+H\left(d x^{+}\right)^{2}+d z_{a}^{2}\right)+e^{2 B} d r^{2}, \quad a=1,2, \cdots, D-3,
$$


in arbitrary dimensions. The functions $A$ and $B$ depend on $r$ only while $H$ depends on $x^{+}, z_{a}$ and $r$ coordinates. If we set $H=0$ the pp-waves reduce to AdS-domain wall solutions [27]. It is natural to choose the following vielbein basis

$$
e^{+}=e^{A} d x^{+}, \quad e^{-}=e^{A}\left(-2 d x^{-}+\frac{1}{2} H d x^{+}\right), \quad e^{a}=e^{A} d z^{a}, \quad e^{r}=e^{B} d r
$$

such that we have $d s^{2}=2 e^{+} e^{-}+e^{a} e^{a}+e^{r} e^{r}$. The vielbein components of the spin connections are

$$
\begin{aligned}
& \omega_{-r}=A^{\prime} e^{-B} e^{+}, \quad \omega_{+a}=\frac{1}{2} e^{-A} \partial_{a} H e^{+}, \\
& \omega_{+r}=A^{\prime} e^{-B} e^{-}+\frac{1}{2} H^{\prime} e^{-B} e^{+}, \quad \omega_{a r}=A^{\prime} e^{-B} e^{a} .
\end{aligned}
$$

where the prime denotes the derivative with respect to $r$. Note that for the metric in this basis we have $\eta_{+-}=1$ and $\eta_{++}=\eta_{--}=0$. The derivatives are always with respect to the curved metric. The vielbein components of the Ricci tensor in $D$-dimensions are given by

$$
\begin{aligned}
R_{++} & =-\frac{1}{2} e^{-2 B}\left[H^{\prime \prime}+H^{\prime}\left((D-1) A^{\prime}-B^{\prime}\right)\right]-\frac{1}{2} e^{-2 A} \sum_{a} \partial_{a} \partial_{a} H=-\frac{1}{2} \square H, \\
R_{+-} & =-e^{-2 B}\left[A^{\prime \prime}+A^{\prime}\left((D-1) A^{\prime}-B^{\prime}\right)\right], \quad R_{a b}=R_{+-} \delta_{a b}, \\
R_{r r} & =-(D-1) e^{-2 B}\left[A^{\prime \prime}+A^{\prime}\left(A^{\prime}-B^{\prime}\right)\right] .
\end{aligned}
$$

It is straightforward to verify that in five dimensions the following

$$
\begin{array}{rlrl}
e^{2 A} & =(g r)^{2}\left[H_{1} H_{2} H_{3}\right]^{1 / 3}, & H_{i}=1+\frac{\ell_{i}^{2}}{r^{2}}, \\
e^{2 B}=\frac{1}{(g r)^{2}\left[H_{1} H_{2} H_{3}\right]^{2 / 3}}, & X_{i}=H_{i}^{-1}\left[H_{1} H_{2} H_{3}\right]^{1 / 3}, \\
A_{(1)}^{i}=g^{-1} S_{i}\left(1-H_{i}^{-1}\right) d x^{+} &
\end{array}
$$

satisfy the equations of motion with $H\left(x^{+}, r, z_{a}\right)$ obeying the equation

$$
H^{\prime \prime}+\left(4 A^{\prime}-B^{\prime}\right) H^{\prime}+e^{-2(A-B)} \sum_{a} \partial_{a} \partial_{a} H+\frac{4 g^{2}}{r^{2}} e^{-6 A} \sum_{i} S_{i}^{2} \ell_{i}^{4} H_{i}^{-2}=0 .
$$

Here the $S_{i}$ are functions of $x^{+}$.

\subsection{Standard supersymmetry}

The Killing spinor equations following from the fermionic transformations are given by

$$
\begin{aligned}
{\left[\partial_{+}+\right.} & \frac{1}{2} A^{\prime} e^{A-B}\left(\Gamma_{+}+\frac{1}{2} H \Gamma_{-}\right)\left(\Gamma_{r}+1\right)-\frac{1}{4} e^{A-B} H^{\prime} \Gamma_{r} \Gamma_{-} \\
& -\frac{1}{4}\left(\partial_{1} H \Gamma_{1}+\partial_{2} H \Gamma_{2}\right) \Gamma_{-}-\frac{\mathrm{i}}{2}\left(\sum_{i} S_{i}\left(1-H_{i}^{-1}\right)\right)\left(\Gamma_{r}+1\right) \\
& \left.+\frac{\mathrm{i}}{6 r^{2}}\left(\sum_{i} S_{i} \ell_{i}^{2} H_{i}^{-1}\right) \Gamma_{r} \Gamma_{+} \Gamma_{-}\right] \epsilon=0
\end{aligned}
$$




$$
\begin{aligned}
& {\left[\partial_{-}-A^{\prime} e^{A-B} \Gamma_{-}\left(\Gamma_{r}+1\right)\right] \epsilon=0,} \\
& {\left[\partial_{a}+\frac{1}{2} A^{\prime} e^{A-B} \Gamma_{a}\left(\Gamma_{r}+1\right)-\frac{\mathrm{i}}{6 r^{2}}\left(\sum_{i} S_{i} \ell_{i}^{2} H_{i}^{-1}\right) \Gamma_{a} \Gamma_{r} \Gamma_{-}\right] \epsilon=0,} \\
& {\left[\partial_{r}+\frac{1}{6 r}\left(\sum_{i} H_{i}^{-1}\right) \Gamma_{r}+\frac{\mathrm{i}}{3 r} g e^{-3 A}\left(\sum_{i} S_{i} \ell_{i}^{2} H_{i}^{-1}\right) \Gamma_{-}\right] \epsilon=0,} \\
& {\left[\mathrm{i} e^{3 A}\left(\sum_{i} a_{1 i} H_{i}^{-1}\right)\left(\Gamma_{r}+1\right)+g\left(\sum_{i} a_{1 i} S_{i} \ell_{i}^{2} H_{i}^{-1}\right) \Gamma_{r} \Gamma_{-}\right] \epsilon=0,} \\
& {\left[\mathrm{i} e^{3 A}\left(\sum_{i} a_{2 i} H_{i}^{-1}\right)\left(\Gamma_{r}+1\right)+g\left(\sum_{i} a_{2 i} S_{i} \ell_{i}^{2} H_{i}^{-1}\right) \Gamma_{r} \Gamma_{-}\right] \epsilon=0,}
\end{aligned}
$$

where we have $\Gamma_{+}^{2}=\Gamma_{-}^{2}=0$ and $\left\{\Gamma_{+}, \Gamma_{-}\right\}=2$. To arrive at these equations we have made use of the solution (10). The above Killing spinor equations have the solution

$$
\epsilon=r^{1 / 2}\left[H_{1} H_{2} H_{3}\right]^{\frac{1}{12}} \epsilon_{0}
$$

where $\epsilon_{0}$ is a constant spinor satisfying $\left(\Gamma_{r}+1\right) \epsilon_{0}=0$ and $\Gamma_{-} \epsilon_{0}=0$. The solution therefore

preserves $\frac{1}{4}$ of the supersymmetry. The Killing spinor for the $\frac{1}{4}$ supersymmetry exist for arbitrary solutions to eq.(11).

\subsection{Supernumerary supersymmetry}

To investigate the supernumerary supersymmetry we use the less restrictive projection condition

$$
\left(\Gamma_{r}+1\right) \epsilon=\mathrm{if} \Gamma_{-} \epsilon
$$

where the function $f=f\left(x^{+}, r, z_{a}\right)$ is to be determined. Making use of this projection in the Killing spinor equations they become

$$
\begin{aligned}
& {\left[\partial_{+}+\frac{\mathrm{i}}{2}\left(A^{\prime} e^{A-B} f-\frac{1}{3 r^{2}} \mathcal{M}\right) \Gamma_{+} \Gamma_{-}+\frac{1}{4 r^{2}}\left(2 f \mathcal{M}-r^{2} e^{A-B} H^{\prime}\right) \Gamma_{-}\right.} \\
& \left.\quad-\frac{1}{4}\left(\partial_{1} H \Gamma_{1}+\partial_{2} H \Gamma_{2}\right) \Gamma_{-}\right] \epsilon=0, \\
& {\left[\partial_{a}+\frac{\mathrm{i}}{2}\left(A^{\prime} e^{A-B} f-\frac{1}{3 r^{2}} \mathcal{M}\right) \Gamma_{a} \Gamma_{-}\right] \epsilon=0, \quad \partial_{-} \epsilon=0,} \\
& {\left[\partial_{r}+\frac{\mathrm{i}}{6 r}\left(f \sum_{i} H_{i}^{-1}+2 g e^{-3 A} \mathcal{M}\right) \Gamma_{-}-\frac{1}{6 r} \sum_{i} H_{i}^{-1}\right] \epsilon=0,} \\
& {\left[e^{3 A} f \sum_{i} a_{b i} H_{i}^{-1}-g \sum_{i} a_{b i} S_{i} \ell_{i}^{2} H_{i}^{-1}\right] \Gamma_{-} \epsilon=0, \quad b=1,2}
\end{aligned}
$$

where $\mathcal{M} \equiv \sum_{i} S_{i} \ell_{i}^{2} H_{i}^{-1}$. We analyse these equations by calculating the integrability conditions $\left[\partial_{M}, \partial_{N}\right] \epsilon=0$ among them. The integrability $\left[\partial_{a}, \partial_{r}\right] \epsilon=0$ yields a solution for $f$ with the requirement $\partial_{a} f=0$. We have

$$
f=\frac{1}{3 r^{2} A^{\prime}} e^{-(A-B)}\left(\mathcal{M}+3 r^{2} U\left(x^{+}\right)\right)
$$


where the function $U$ is in general complex. From the integrability $\left[\partial_{+}, \partial_{a}\right] \epsilon=0$ we obtain an equation for $U$ after imposing some restrictions on the pp-wave function $H\left(x^{+}, r, z_{a}\right)$. The result is

$$
\begin{aligned}
& \partial_{a} H^{\prime}=0, \quad \partial_{a} \partial_{b} H=0 \quad \text { for } \quad a \neq b, \\
& \mathrm{i} \frac{d U}{d x^{+}}+U^{2}+\frac{1}{2} \partial_{a} \partial_{a} H=0, \quad a=1,2 .
\end{aligned}
$$

The equation for $U$ then requires $\partial_{1} \partial_{1} H=\partial_{2} \partial_{2} H$. Investigating the pair of equations given in the last line of eqs.(15) we find that they are satisfied provided that the functions $S_{i}$ and $U$ satisfy two equations among them. We present the solutions in terms of $S_{3}$ and $U$. They are given by

$$
S_{1}=\ell_{1}^{-2}\left(S_{3} \ell_{3}^{2}-\left(\ell_{1}^{2}-\ell_{3}^{2}\right) U\right), \quad S_{2}=\ell_{2}^{-2}\left(S_{3} \ell_{3}^{2}-\left(\ell_{2}^{2}-\ell_{3}^{2}\right) U\right)
$$

In order to analyse the final integrability $\left[\partial_{+}, \partial_{r}\right] \epsilon=0$ we need to make use of the solution for $H$. Taking into account the conditions on $H$ given above the solution is given by

$$
\begin{aligned}
g^{4} H\left(x^{+}, r, z_{a}\right)= & \frac{1}{2} c g^{4}\left(z_{1}^{2}+z_{2}^{2}\right)+\frac{1}{2}\left|\epsilon_{i j k}\right| K_{i j k}\left(x^{+}, r\right), \\
K_{i j k}\left(x^{+}, r\right)= & -\frac{S_{i}^{2} \ell_{i}^{4}}{\left(\ell_{i}^{2}-\ell_{j}^{2}\right)\left(\ell_{i}^{2}-\ell_{k}^{2}\right)\left(r^{2}+\ell_{i}^{2}\right)} \\
& +\frac{1}{2\left(\ell_{i}^{2}-\ell_{j}^{2}\right)^{2}\left(\ell_{i}^{2}-\ell_{k}^{2}\right)^{2}}\left[\left(b g^{4}+c \ell_{i}^{2}\right)\left(\ell_{i}^{2}-\ell_{j}^{2}\right)\left(\ell_{i}^{2}-\ell_{k}^{2}\right)\right. \\
& \left.+2 S_{i}^{2} \ell_{i}^{4}\left(2 \ell_{i}^{2}-\ell_{j}^{2}-\ell_{k}^{2}\right)-2 S_{j}^{2} \ell_{j}^{4}\left(\ell_{i}^{2}-\ell_{k}^{2}\right)-2 S_{k}^{2} \ell_{k}^{4}\left(\ell_{i}^{2}-\ell_{j}^{2}\right)\right] \ln \left(r^{2}+\ell_{i}^{2}\right),
\end{aligned}
$$

where $b=b\left(x^{+}\right)$and $c=c\left(x^{+}\right)$. Then $\left[\partial_{+}, \partial_{r}\right] \epsilon=0$ yields an equation for $S_{3}$ given by

$$
\mathrm{i} \frac{d S_{3}}{d x^{+}}-\left(2 \ell_{3}\right)^{-2}\left[b g^{4}+c \ell_{3}^{2}+2 U\left(U\left(\ell_{1}^{2}+\ell_{2}^{2}\right)-2 \ell_{3}^{2}\left(2 S_{3}+U\right)\right)\right]=0 .
$$

We proceed next by making use of the information that $S_{i}, b$ and $c$ are real functions. Eqs.(18) implies that $U$ must also be real. This has the consequence in eq. (17) that $U$ and $c$ must be constants with $c$ being given by $c=-2 U^{2}$. Eqs.(201) and (18) in turn implies that $S_{i}$ and $b$ must also be constants. Eliminating $U$ from eqs.(18) and setting $S_{i}=\mu_{i}$ we obtain

$$
\epsilon_{i j k} \mu_{i} \ell_{i}^{2}\left(\ell_{j}^{2}-\ell_{k}^{2}\right)=0
$$

Without loss of generality we solve for $\mu_{1}$ in terms of the other two charges. The function $H$ which gives $\frac{1}{2}$ supersymmetric pp-wave is given by

$$
\mu_{1}=\frac{\mu_{2} \ell_{2}^{2}\left(\ell_{1}^{2}-\ell_{3}^{2}\right)-\mu_{3} \ell_{3}^{2}\left(\ell_{1}^{2}-\ell_{2}^{2}\right)}{\ell_{1}^{2}\left(\ell_{2}^{2}-\ell_{3}^{2}\right)}
$$




$$
\begin{aligned}
b & =-\frac{2\left(\mu_{2} \ell_{2}^{2}-\mu_{3} \ell_{3}^{2}\right)\left(\mu_{2} \ell_{2}^{4}-\mu_{3} \ell_{3}^{4}-3\left(\mu_{2}-\mu_{3}\right) \ell_{2}^{2} \ell_{3}^{2}+\ell_{1}^{2}\left(\mu_{2} \ell_{2}^{2}-\mu_{3} \ell_{3}^{2}\right)\right)}{g^{4}\left(\ell_{2}^{2}-\ell_{3}^{2}\right)^{2}} \\
c & =-\frac{2\left(\mu_{2} \ell_{2}^{2}-\mu_{3} \ell_{3}^{2}\right)^{2}}{\left(\ell_{2}^{2}-\ell_{3}^{2}\right)^{2}} \\
H & =\frac{1}{2} c\left(z_{1}^{2}+z_{2}^{2}\right)-f^{2} \\
f & =-\frac{\left(\mu_{2} \ell_{2}^{2}-\mu_{3} \ell_{3}^{2}\right) r^{2}+\left(\mu_{2}-\mu_{3}\right) \ell_{2}^{2} \ell_{3}^{2}}{g^{2}\left(\ell_{2}^{2}-\ell_{3}^{2}\right) r^{3}\left[H_{1} H_{2} H_{3}\right]^{1 / 2}}
\end{aligned}
$$

We next calculate the Killing spinor. The projected Killing spinor equations become

$$
\begin{aligned}
& {\left[\partial_{+}-\frac{1}{2 \sqrt{2}}(-c)^{1 / 2}\left(\mathrm{i} \Gamma_{+}-f\right) \Gamma_{-}-\frac{1}{4} c\left(z_{1} \Gamma_{1}+z_{2} \Gamma_{2}\right) \Gamma_{-}\right] \epsilon=0,} \\
& {\left[\partial_{a}-\frac{\mathrm{i}}{2 \sqrt{2}}(-c)^{1 / 2} \Gamma_{a} \Gamma_{-}\right] \epsilon=0, \quad \partial_{-} \epsilon=0,} \\
& {\left[\partial_{r}-\frac{\mathrm{i}}{2} f^{\prime} \Gamma_{-}-\frac{1}{6 r} \sum_{i} H_{i}^{-1}\right] \epsilon=0 .}
\end{aligned}
$$

The Killing spinor is easily obtained, given by

$$
\begin{aligned}
\epsilon & =r^{1 / 2}\left[H_{1} H_{2} H_{3}\right]^{\frac{1}{12}}\left(1+\frac{\mathrm{i}}{2 \sqrt{2}}(-c)^{1 / 2}\left(z_{1} \Gamma_{1}+z_{2} \Gamma_{2}\right) \Gamma_{-}\right)\left(1+\frac{\mathrm{i}}{2} f \Gamma_{-}\right) \eta, \\
\frac{d \eta}{d x^{+}} & =\frac{\mathrm{i}}{2 \sqrt{2}}(-c)^{1 / 2} \Gamma_{+} \Gamma_{-} \eta .
\end{aligned}
$$

Solving for $\eta$ we have

$$
\begin{gathered}
\epsilon=r^{1 / 2}\left[H_{1} H_{2} H_{3}\right]^{\frac{1}{12}}\left(1+\frac{\mathrm{i}}{2 \sqrt{2}}(-c)^{1 / 2}\left(z_{1} \Gamma_{1}+z_{2} \Gamma_{2}\right) \Gamma_{-}\right)\left(1+\frac{\mathrm{i}}{2} f \Gamma_{-}\right) \\
\times\left[1-\frac{1}{2}\left(1-e^{\frac{\mathrm{i}}{\sqrt{2}}(-c)^{1 / 2} x^{+}}\right) \Gamma_{+} \Gamma_{-}\right] \epsilon_{0}
\end{gathered}
$$

where $\epsilon_{0}$ is a constant spinor satisfying $\left(\Gamma_{r}+1\right) \epsilon_{0}=0$. The solution thus preserve $\frac{1}{2}$ of the supersymmetry. Note that if we set $\mu_{i} \ell_{i}^{2}=\mu$ (which is consistent with eq.(21) ) we obtain $b=c=0$.

To conclude, demanding supernumerary supersymmetry puts very strong restrictions on the pp-waves with the functions $S, b$ and $c$ (and $U$ ) which initially all being functions of $x^{+}$reduce now to constants. This is not the case for minimal gauged supergravity where supernumerary supersymmetry does allow the various functions to have $x^{+}$dependence.

\section{PP-waves in four dimensions}

In this section we consider a subsector of the $S O(8)$ gauged supergravity where the bosonic fields comprises the metric, four commuting $U(1)$ gauge potentials and three dilatons. The Lagrangian describing this set of fields is 28

$$
e^{-1} \mathcal{L}_{4}=R-\frac{1}{2}(\partial \vec{\varphi})^{2}-\frac{1}{4} \sum_{i} X_{i}^{-2}\left(F_{(2)}^{i}\right)^{2}-V,
$$


where $\vec{\varphi}=\left(\varphi_{1}, \varphi_{2}, \varphi_{3}\right)$, and

$$
\begin{aligned}
& X_{i}=e^{-\frac{1}{2} \vec{a}_{i} \cdot \vec{\varphi}}, \quad X_{1} X_{2} X_{3} X_{4}=1, \\
& \vec{a}_{1}=(1,1,1), \quad \vec{a}_{2}=(1,-1,-1), \quad \vec{a}_{3}=(-1,1,-1), \quad \vec{a}_{3}=(-1,-1,1) .
\end{aligned}
$$

The field strengths are defined as $F_{(2)}^{i}=d A_{(1)}^{i}$ and the potential is given by

$$
V=-4 g^{2} \sum_{i<j} X_{i} X_{j}=-8 g^{2} \sum_{i=1}^{3} \cosh \varphi_{i}
$$

The $\mathcal{N}=8$ supersymmetry transformations in this bosonic background were also presented in [28. They are given by

$$
\begin{aligned}
\delta \Psi_{M}^{i} & =\nabla_{M} \epsilon^{(i)}+\sum_{j}\left[-\mathrm{i} g \Omega_{i j} A_{M}^{j}+\frac{\mathrm{i}}{8} \Omega_{i j} X_{j}^{-1} F_{A B}^{j} \Gamma^{A B} \Gamma_{M}+\frac{1}{4} g X_{j} \Gamma_{M}\right] \epsilon^{(i)} \\
\delta \lambda^{i j} & =\left[\frac{\mathrm{i}}{\sqrt{2}} \Gamma^{M} \partial_{M} \phi^{i j}-\frac{1}{2 \sqrt{2}} \sum_{k} \Omega_{j k} X_{k}^{-1} F_{A B}^{k} \Gamma^{A B}+\mathrm{i} \sqrt{2} g \sum_{k, m} f_{i j k} \Omega_{k m} X_{m}\right] \epsilon^{(i)},
\end{aligned}
$$

where we have rewritten them by introducing complex fermions $\Psi_{M}^{i}=\Psi_{1 M}^{i}+\mathrm{i} \Psi_{2 M}^{i}$, etc and made the substitutions $g \rightarrow \sqrt{2} g$ and $A_{(1)}^{i} \rightarrow-\frac{1}{2 \sqrt{2}} A_{(1)}^{i}$. Note that $i \neq j$ in the spin $1 / 2$ transformations. The three dilatons are given by the following identifications

$$
\varphi_{1}=\phi^{12}=\phi^{34}, \quad \varphi_{2}=\phi^{13}=\phi^{24}, \quad \varphi_{3}=\phi^{14}=\phi^{23}
$$

and note also that $\phi^{i j}=\phi^{j i}$. The function $f_{i j k}$ is defined as

$$
f_{i j k}=\left\{\begin{aligned}
\left|\epsilon_{i j k}\right| & \text { for } \quad i, j, k \neq 1 \\
\delta_{j k} & \text { for } \quad i=1 \\
\delta_{i k} & \text { for } \quad j=1
\end{aligned}\right.
$$

and the matrix $\Omega$ is given by

$$
\Omega=\frac{1}{2}\left(\begin{array}{rrrr}
1 & 1 & 1 & 1 \\
1 & 1 & -1 & -1 \\
1 & -1 & 1 & -1 \\
1 & -1 & -1 & 1
\end{array}\right)
$$

\subsection{The solution}

The four charge pp-wave is given by

$$
d s_{4}^{2}=e^{2 A}\left(-4 d x^{+} d x^{-}+H\left(d x^{+}\right)^{2}+d z^{2}\right)+e^{2 B} d r^{2},
$$


where

$$
\begin{array}{rlrl}
e^{2 A} & =(g r)^{4}\left[H_{1} H_{2} H_{3} H_{4}\right]^{1 / 2}, & H_{i}=1+\frac{\ell_{i}^{2}}{r^{2}}, \\
e^{2 B} & =\frac{1}{(g r)^{2}\left[H_{1} H_{2} H_{3} H_{4}\right]^{1 / 2}}, & & X_{i}=H_{i}^{-1}\left[H_{1} H_{2} H_{3} H_{4}\right]^{1 / 4}, \\
A_{(1)}^{i} & =g^{-1} S_{i}\left(1-H_{i}^{-1}\right) d x^{+} &
\end{array}
$$

and $S_{i}=S_{i}\left(x^{+}\right)$. The function $H\left(x^{+}, r, z\right)$ satisfies the equation

$$
H^{\prime \prime}+\left(3 A^{\prime}-B^{\prime}\right) H^{\prime}+e^{-2(A-B)} \partial_{z} \partial_{z} H+\frac{4 g^{2}}{r^{2}} e^{-4 A} \sum_{i} S_{i}^{2} \ell_{i}^{4} H_{i}^{-2}=0 .
$$

The solution to this equation is similar to the solution in $D=5$. The four charged pp-wave can be specialised to one, two and three active charges respectively.

\subsection{Supersymmetry}

The $\mathcal{N}=8$ supersymmetry have four different sectors. We begin by analysing the Killing spinor equations for the sector $\epsilon^{(1)}$. The supersymmetry transformations are given by

$$
\begin{aligned}
& \delta \Psi_{M}^{1}=\nabla_{M} \epsilon^{(1)}+\sum_{i=1}^{4}\left[-\frac{\mathrm{i}}{2} g A_{M}^{i}+\frac{\mathrm{i}}{16} X_{i}^{-1} F_{A B}^{i} \Gamma^{A B} \Gamma_{M}+\frac{1}{4} g X_{i} \Gamma_{M}\right] \epsilon^{(1)}, \\
& \delta \lambda^{12}=\left[\frac{\mathrm{i}}{\sqrt{2}} \Gamma^{M} \partial_{M} \varphi_{1}-\frac{1}{4 \sqrt{2}} \Gamma^{A B}\left(X_{1}^{-1} F_{A B}^{1}+X_{2}^{-1} F_{A B}^{2}-X_{3}^{-1} F_{A B}^{3}-X_{4}^{-1} F_{A B}^{4}\right)\right. \\
& \left.+\frac{\mathrm{i}}{\sqrt{2}} g\left(X_{1}+X_{2}-X_{3}-X_{4}\right)\right] \epsilon^{(1)}, \\
& \delta \lambda^{13}=\left[\frac{\mathrm{i}}{\sqrt{2}} \Gamma^{M} \partial_{M} \varphi_{2}-\frac{1}{4 \sqrt{2}} \Gamma^{A B}\left(X_{1}^{-1} F_{A B}^{1}-X_{2}^{-1} F_{A B}^{2}+X_{3}^{-1} F_{A B}^{3}-X_{4}^{-1} F_{A B}^{4}\right)\right. \\
& \left.+\frac{\mathrm{i}}{\sqrt{2}} g\left(X_{1}-X_{2}+X_{3}-X_{4}\right)\right] \epsilon^{(1)}, \\
& \delta \lambda^{14}=\left[\frac{\mathrm{i}}{\sqrt{2}} \Gamma^{M} \partial_{M} \varphi_{3}-\frac{1}{4 \sqrt{2}} \Gamma^{A B}\left(X_{1}^{-1} F_{A B}^{1}-X_{2}^{-1} F_{A B}^{2}-X_{3}^{-1} F_{A B}^{3}+X_{4}^{-1} F_{A B}^{4}\right)\right. \\
& \left.+\frac{\mathrm{i}}{\sqrt{2}} g\left(X_{1}-X_{2}-X_{3}+X_{4}\right)\right] \epsilon^{(1)} \text {. }
\end{aligned}
$$

The Killing spinor equations are readily written down and take the form

$$
\begin{aligned}
& {\left[\partial_{+}+\frac{1}{2} A^{\prime} e^{A-B}\left(\Gamma_{+}+\frac{1}{2} H \Gamma_{-}\right)\left(\Gamma_{r}+1\right)-\frac{1}{4} H^{\prime} e^{A-B} \Gamma_{r} \Gamma_{-}-\frac{1}{4} \partial_{z} H \Gamma_{z} \Gamma_{-}\right.} \\
& \left.\quad-\frac{\mathrm{i}}{2}\left(\sum_{i=1}^{4} S_{i}\left(1-H_{i}^{-1}\right)\right)\left(\Gamma_{r}+1\right)+\frac{\mathrm{i}}{4 r^{2}}\left(\sum_{i} \mathcal{M}_{i}\right) \Gamma_{r} \Gamma_{+} \Gamma_{-}\right] \epsilon^{(1)}=0 \\
& {\left[\partial_{-}-A^{\prime} e^{A-B} \Gamma_{-}\left(\Gamma_{r}+1\right)\right] \epsilon^{(1)}=0} \\
& {\left[\partial_{z}+\frac{1}{2} A^{\prime} e^{A-B} \Gamma_{z}\left(\Gamma_{r}+1\right)-\frac{\mathrm{i}}{4 r^{2}}\left(\sum_{i} \mathcal{M}_{i}\right) \Gamma_{z} \Gamma_{r} \Gamma_{-}\right] \epsilon^{(1)}=0} \\
& {\left[\partial_{r}+\frac{1}{4 r}\left(\sum_{i} H_{i}^{-1}\right) \Gamma_{r}+\frac{\mathrm{i}}{4 r} g e^{-2 A}\left(\sum_{i} \mathcal{M}_{i}\right) \Gamma_{-}\right] \epsilon^{(1)}=0}
\end{aligned}
$$




$$
\begin{aligned}
& {\left[\mathrm{i} g\left(X_{1}+X_{2}-X_{3}-X_{4}\right)\left(\Gamma_{r}+1\right)+\frac{1}{r^{2}} e^{-A}\left(\mathcal{M}_{1}+\mathcal{M}_{2}-\mathcal{M}_{3}-\mathcal{M}_{4}\right) \Gamma_{r} \Gamma_{-}\right] \epsilon^{(1)}=0,} \\
& {\left[\mathrm{i} g\left(X_{1}-X_{2}+X_{3}-X_{4}\right)\left(\Gamma_{r}+1\right)+\frac{1}{r^{2}} e^{-A}\left(\mathcal{M}_{1}-\mathcal{M}_{2}+\mathcal{M}_{3}-\mathcal{M}_{4}\right) \Gamma_{r} \Gamma_{-}\right] \epsilon^{(1)}=0,} \\
& {\left[\mathrm{i} g\left(X_{1}-X_{2}-X_{3}+X_{4}\right)\left(\Gamma_{r}+1\right)+\frac{1}{r^{2}} e^{-A}\left(\mathcal{M}_{1}-\mathcal{M}_{2}-\mathcal{M}_{3}+\mathcal{M}_{4}\right) \Gamma_{r} \Gamma_{-}\right] \epsilon^{(1)}=0,}
\end{aligned}
$$

where we have defined $\mathcal{M}_{i} \equiv S_{i} \ell_{i}^{2} H_{i}^{-1}$. These equations have the solution

$$
\epsilon^{(1)}=r\left[H_{1} H_{2} H_{3} H_{4}\right]^{\frac{1}{8}} \epsilon_{0}^{(1)}
$$

where $\epsilon_{0}^{(1)}$ is a constant spinor satisfying $\left(\Gamma_{r}+1\right) \epsilon_{0}^{(1)}=0=\Gamma_{-} \epsilon_{0}^{(1)}$. Thus $\frac{1}{4}$ of the supersymmetry of the $\epsilon^{(1)}$ sector is preserved (standard supersymmetry). It is easy to see that the same amount of supersymmetry is preserved simultaneously in the other sectors. The pp-wave therefore preserves overall $\frac{1}{4}$ of the $\mathcal{N}=8$ supersymmetry.

Now let us examine whether the solution admits supernumerary supersymmetry. We again make use of the ansatz

$$
\left(\Gamma_{r}+1\right) \epsilon^{(1)}=\mathrm{i} f_{1} \Gamma_{-} \epsilon^{(1)} .
$$

A similar analysis of the integrability conditions among the projected Killing spinor equations as in five dimensions shows that the functions $S_{i}\left(x^{+}\right), U\left(x^{+}\right), b\left(x^{+}\right)$and $c\left(x^{+}\right)$must again be constants. In $D=4$ there are now two conditions that must be satisfied among the charges for there to be supernumerary supersymmetry. Hence the pp-wave solution will depend on just two charge parameters. The constraints among the charges are given by

$$
\begin{aligned}
& \ell_{1}^{2} \ell_{4}^{2}\left(\ell_{2}^{2}-\ell_{3}^{2}\right)\left(\mu_{1}-\mu_{4}\right)=\ell_{2}^{2} \ell_{3}^{2}\left(\ell_{1}^{2}-\ell_{4}^{2}\right)\left(\mu_{2}-\mu_{3}\right), \\
& \left(\ell_{2}^{2}-\ell_{3}^{2}\right)\left(\mu_{1} \ell_{1}^{2}-\mu_{4} \ell_{4}^{2}\right)=\left(\ell_{1}^{2}-\ell_{4}^{2}\right)\left(\mu_{2} \ell_{2}^{2}-\mu_{3} \ell_{3}^{2}\right),
\end{aligned}
$$

where we have set $S_{i}=\mu_{i}$. Solving for $\mu_{1}$ and $\mu_{2}$ in terms of the other two charges the function $H$ is given by

$$
\begin{aligned}
\mu_{\alpha} & =\frac{\mu_{3} \ell_{3}^{2}\left(\ell_{\alpha}^{2}-\ell_{4}^{2}\right)-\mu_{4} \ell_{4}^{2}\left(\ell_{\alpha}^{2}-\ell_{3}^{2}\right)}{\ell_{\alpha}^{2}\left(\ell_{3}^{2}-\ell_{4}^{2}\right)}, \quad \alpha=1,2 \\
b & =-\frac{2\left(\mu_{3} \ell_{3}^{2}-\mu_{4} \ell_{4}^{2}\right)}{g^{6}\left(\ell_{3}^{2}-\ell_{4}^{2}\right)^{2}}\left[\mu_{3} \ell_{3}^{2}\left(\ell_{1}^{2}+\ell_{2}^{2}+\ell_{3}^{2}-5 \ell_{4}^{2}\right)-\mu_{4} \ell_{4}^{2}\left(\ell_{1}^{2}+\ell_{2}^{2}-5 \ell_{3}^{2}+\ell_{4}^{2}\right)\right] \\
c & =-\frac{8\left(\mu_{3} \ell_{3}^{2}-\mu_{4} \ell_{4}^{2}\right)^{2}}{\left(\ell_{3}^{2}-\ell_{4}^{2}\right)^{2}} \\
H & =\frac{1}{2} c z^{2}-f_{1}^{2} \\
f_{1} & =-\frac{\left(\mu_{3} \ell_{3}^{2}-\mu_{4} \ell_{4}^{2}\right) r^{2}+\left(\mu_{3}-\mu_{4}\right) \ell_{3}^{2} \ell_{4}^{2}}{g^{3}\left(\ell_{3}^{2}-\ell_{4}^{2}\right) r^{4}\left[H_{1} H_{2} H_{3} H_{4}\right]^{1 / 2}}
\end{aligned}
$$


The projected Killing spinor equations are given by

$$
\begin{aligned}
& {\left[\partial_{+}-\frac{1}{2 \sqrt{2}}(-c)^{1 / 2}\left(\mathrm{i} \Gamma_{+}-f_{1}\right) \Gamma_{-}-\frac{1}{4} c z \Gamma_{z} \Gamma_{-}\right] \epsilon^{(1)}=0,} \\
& {\left[\partial_{z}-\frac{\mathrm{i}}{2 \sqrt{2}}(-c)^{1 / 2} \Gamma_{z} \Gamma_{-}\right] \epsilon^{(1)}=0, \quad \partial_{-} \epsilon^{(1)}=0,} \\
& {\left[\partial_{r}-\frac{\mathrm{i}}{2} f_{1}^{\prime} \Gamma_{-}-\frac{1}{4 r} \sum_{i} H_{i}^{-1}\right] \epsilon^{(1)}=0 .}
\end{aligned}
$$

The solution for the Killing spinor is

$$
\begin{gathered}
\epsilon^{(1)}=r\left[H_{1} H_{2} H_{3} H_{4}\right]^{\frac{1}{8}}\left(1+\frac{\mathrm{i}}{2 \sqrt{2}}(-c)^{1 / 2} z \Gamma_{z} \Gamma_{-}\right)\left(1+\frac{\mathrm{i}}{2} f_{1} \Gamma_{-}\right) \\
\times\left[1-\frac{1}{2}\left(1-e^{\frac{\mathrm{i}}{\sqrt{2}}(-c)^{1 / 2} x^{+}}\right) \Gamma_{+} \Gamma_{-}\right] \epsilon_{0}^{(1)}
\end{gathered}
$$

where $\epsilon_{0}^{(1)}$ is a constant spinor satisfying $\left(\Gamma_{r}+1\right) \epsilon_{0}^{(1)}=0$. The pp-wave with $H$ given above therefore preserves $\frac{1}{2}$ of the supersymmetry of the $\epsilon^{(1)}$ sector. Consider next the remaining sectors. For this we use the ansatz $A_{(1)}^{i}=g^{-1} \eta_{i} \mu_{i}\left(1-H_{i}^{-1}\right) d x^{+}$. To preserve $\frac{1}{2}$ supersymmetry in the four respective sectors then requires the sign choices:

$$
\begin{array}{rrrr}
1: & \eta_{1}=\eta_{2}=\eta_{3}=\eta_{4} \\
2: & \eta_{1}=\eta_{2}=-\eta_{3}=-\eta_{4} \\
3: & \eta_{1}=-\eta_{2}=\eta_{3}=-\eta_{4} \\
4: & \eta_{1}=-\eta_{2}=-\eta_{3}=\eta_{4}
\end{array}
$$

Because of the difference in signs the four charge solution will preserve $\frac{1}{2}$ of the supersymmetry of just one sector and $\frac{1}{4}$ of the supersymmetry of each of the remaining sectors.

Although we have focused on solutions with four active charges one can easily also analyse the supersymmetry of solutions with one, two or three active charges. In the following table we present the overall amount of the $\mathcal{N}=8$ supersymmetry preserved in the various cases.

\begin{tabular}{|c|c|c|}
\hline $\begin{array}{c}\text { No. of active } \\
\text { charges }\end{array}$ & $\begin{array}{c}\text { Standard } \\
\text { supersymmetry }\end{array}$ & $\begin{array}{c}\text { Enhanced } \\
\text { supersymmetry }\end{array}$ \\
\hline \hline 1 & $\frac{1}{4}$ & $\frac{1}{8}+\frac{1}{8}+\frac{1}{8}+\frac{1}{8}=\frac{1}{2}$ \\
\hline 2 & $\frac{1}{4}$ & $\frac{1}{8}+\frac{1}{8}+\frac{1}{16}+\frac{1}{16}=\frac{3}{8}$ \\
\hline 3 & $\frac{1}{4}$ & $\frac{1}{8}+\frac{1}{16}+\frac{1}{16}+\frac{1}{16}=\frac{5}{16}$ \\
\hline 4 & $\frac{1}{4}$ & $\frac{1}{8}+\frac{1}{16}+\frac{1}{16}+\frac{1}{16}=\frac{5}{16}$ \\
\hline
\end{tabular}

Table 1: Amount of $\mathcal{N}=8$ supersymmetry preserved by $1,2,3$ and 4 active charged pp-waves. 


\section{PP-waves in the Freedman-Schwarz model}

The Lagrangian describing the bosonic sector of the Freedman-Schwarz model is [29]

$$
\begin{array}{rl}
\mathcal{L}_{4}=R & * \mathbb{1}-\frac{1}{2} * d \phi \wedge d \phi-\frac{1}{2} e^{2 \phi} * d \chi \wedge d \chi+4\left(g_{1}^{2}+g_{2}^{2}\right) e^{\phi} * \mathbb{1} \\
& -\frac{1}{2} e^{-\phi}\left(* F_{(2)}^{a} \wedge F_{(2)}^{a}+* G_{(2)}^{a} \wedge G_{(2)}^{a}\right)-\frac{1}{2} \chi\left(F_{(2)}^{a} \wedge F_{(2)}^{a}+G_{(2)}^{a} \wedge G_{(2)}^{a}\right),
\end{array}
$$

where

$$
\begin{aligned}
F_{(2)}^{a} & =d A_{(1)}^{a}-\frac{1}{\sqrt{2}} g_{1} \epsilon_{a b c} A_{(1)}^{b} \wedge A_{(1)}^{c}, \quad a=1,2,3 \\
G_{(2)}^{a} & =d B_{(1)}^{a}-\frac{1}{\sqrt{2}} g_{2} \epsilon_{a b c} B_{(1)}^{b} \wedge B_{(1)}^{c} .
\end{aligned}
$$

The supersymmetry transformations for the fermions are given by

$$
\begin{aligned}
\delta \Psi_{M}= & {\left[\nabla_{M}-\frac{\mathrm{i}}{\sqrt{2}} g_{1} \alpha_{1}^{a} A_{M}^{a}-\frac{\mathrm{i}}{\sqrt{2}} g_{2} \alpha_{2}^{a} B_{M}^{a}-\frac{\mathrm{i}}{4} e^{\phi} \Gamma_{5} \partial_{M} \chi\right.} \\
& \left.+\frac{\mathrm{i}}{8 \sqrt{2}} e^{-\frac{1}{2} \phi}\left(\alpha_{1}^{a} F_{A B}^{a}-\mathrm{i} \Gamma_{5} \alpha_{2}^{a} G_{A B}^{a}\right) \Gamma^{A B} \Gamma_{M}+\frac{1}{2} e^{\frac{1}{2} \phi}\left(g_{1}-\mathrm{i} g_{2} \Gamma_{5}\right) \Gamma_{M}\right] \epsilon, \\
\delta \lambda= & {\left[\frac{\mathrm{i}}{\sqrt{2}}\left(\partial_{M} \phi-\mathrm{i} e^{\phi} \Gamma_{5} \partial_{M} \chi\right) \Gamma^{M}+\frac{1}{4} e^{-\frac{1}{2} \phi}\left(\alpha_{1}^{a} F_{A B}^{a}+\mathrm{i} \Gamma_{5} \alpha_{2}^{a} G_{A B}^{a}\right) \Gamma^{A B}\right.} \\
& \left.\quad-\mathrm{i} \sqrt{2} e^{\frac{1}{2} \phi}\left(g_{1}+\mathrm{i} g_{2} \Gamma_{5}\right)\right] \epsilon,
\end{aligned}
$$

where $\Gamma_{5}=\mathrm{i} \Gamma_{0} \Gamma_{1} \Gamma_{2} \Gamma_{3}$ such that $\Gamma_{5}^{2}=1$. The $\alpha_{1}^{a}$ and $\alpha_{2}^{a}$ are two sets Pauli matrices. The gravitino, the dilatino and the (Majorana) spinor $\epsilon$ carry a suppressed indice which runs from one to four. In the following we turn off two of the fields $F_{M N}^{a}$ and $G_{M N}^{a}$ each. For a vanishing axion $(\chi=0)$ the pp-wave in this theory is given by

$$
\begin{aligned}
d s^{2} & =(g r)^{2}\left(-4 d x^{+} d x^{-}+H\left(d x^{+}\right)^{2}+d z^{2}\right)+d r^{2} \\
H & =\frac{1}{2} c z^{2}-\frac{b}{(g r)^{2}}-\frac{c \ln (g r)}{2 g^{2}}-\frac{g_{2}^{2} S_{1}^{2}+g_{1}^{2} S_{2}^{2}}{2 g_{1}^{2} g_{2}^{2}(g r)^{4}} \\
\phi & =-2 \ln (g r) \\
A_{(1)} & =g_{1}^{-1} S_{1}\left(x^{+}\right)\left((g r)^{-2}-1\right) d x^{+}, \quad B_{(1)}=g_{2}^{-1} S_{2}\left(x^{+}\right)\left((g r)^{-2}-1\right) d x^{+},
\end{aligned}
$$

where $g=\left(g_{1}^{2}+g_{2}^{2}\right)^{1 / 2}$. Now lets look at the supersymmetry of this solution. The Killing spinor equations are given by

$$
\begin{aligned}
& {\left[\partial_{+}+\frac{1}{2} g\left(\Gamma_{+}+\frac{1}{2} H \Gamma_{-}\right)\left(\Gamma_{r}+a\right)-\frac{\mathrm{i}}{\sqrt{2}}\left(S_{1}+S_{2}\right)\left((g r)^{-2}-1\right)\right.} \\
& \left.\quad-\frac{1}{4} c z \Gamma_{z} \Gamma_{-}-\frac{1}{4} g r H^{\prime} \Gamma_{r} \Gamma_{-}-\mathrm{i} \Lambda \Gamma_{-} \Gamma_{+} \Gamma_{r}\right] \epsilon=0, \\
& {\left[\partial_{-}-g \Gamma_{-}\left(\Gamma_{r}+a\right)\right] \epsilon=0,} \\
& {\left[\partial_{z}+\frac{1}{2} g \Gamma_{z}\left(\Gamma_{r}+a\right)+\mathrm{i} \Lambda \Gamma_{z} \Gamma_{-} \Gamma_{r}\right] \epsilon=0,} \\
& {\left[\partial_{r}+\frac{\mathrm{i} \Lambda}{g r} \Gamma_{-}+\frac{1}{2 g r}\left(g_{1}-\mathrm{i} g_{2} \Gamma_{5}\right) \Gamma_{r}\right] \epsilon=0,} \\
& {\left[\left(\Gamma_{r}+a\right)+2 \mathrm{i} g^{-1} \Gamma_{-} \Lambda \Gamma_{r}\right] \epsilon=0,}
\end{aligned}
$$


where we have defined

$$
a=g^{-1}\left(g_{1}+\mathrm{i} g_{2} \Gamma_{5}\right) \quad \text { and } \quad \Lambda=\frac{g_{2} S_{1}-\mathrm{i} g_{1} S_{2} \Gamma_{5}}{2 \sqrt{2} g_{1} g_{2}\left(g_{1}^{2}+g_{2}^{2}\right)^{1 / 2} r^{2}}
$$

It follows from these equations that to obtain the usual $\frac{1}{4}$ supersymmetry for the pp-wave we need surprisingly to impose $g_{2}^{2} S_{1}=g_{1}^{2} S_{2}$. The Killing spinor can then be obtained and it is given by

$$
\epsilon=e^{-\frac{\mathrm{i}}{\sqrt{2}} \int\left(S_{1}+S_{2}\right) d x^{+}} r^{1 / 2} \epsilon_{0}
$$

where $\epsilon_{0}$ is a constant spinor satisfying the projections $\left(\Gamma_{r}+a\right) \epsilon_{0}=0=\Gamma_{-} \epsilon_{0}$. To investigate the supernumerary supersymmetry we use the projection condition

$$
\left(\Gamma_{r}+a\right) \epsilon=\mathrm{if} \Gamma_{-} \epsilon
$$

The projected Killing spinor equations are given by

$$
\begin{aligned}
& {\left[\partial_{+}+\frac{\mathrm{i}}{\sqrt{2}}\left(S_{1}+S_{2}\right)+\mathrm{i} \Gamma_{+}\left(\frac{1}{2} g f-\bar{a} \bar{\Lambda}\right) \Gamma_{-}-\frac{1}{4} c z \Gamma_{z} \Gamma_{-}\right.} \\
& \left.\quad-\frac{1}{2 \sqrt{2} g_{1} g_{2} g^{2} r^{2}}\left(S_{1} g_{2}^{2}-S_{2} g_{1}^{2}\right) \Gamma_{5}+\left(2 \Lambda f-\frac{1}{4} a g r H^{\prime}\right) \Gamma_{-}\right] \epsilon=0, \\
& {\left[\partial_{z}+\mathrm{i} \Gamma_{z}\left(\frac{1}{2} g f-\bar{a} \bar{\Lambda}\right) \Gamma_{-}\right] \epsilon=0, \quad \partial_{-} \epsilon=0,} \\
& {\left[\partial_{r}+\frac{\mathrm{i}}{r}\left(g^{-1} \Lambda+\frac{1}{2} \bar{a} f\right) \Gamma_{-}-\frac{1}{2 r}\right] \epsilon=0,} \\
& {\left[f-2 g^{-1} \bar{a} \bar{\Lambda}\right] \Gamma_{-} \epsilon=0 .}
\end{aligned}
$$

Here $\bar{a}$ and $\bar{\Lambda}$ are just $a$ and $\Lambda$ but with $\Gamma_{5}$ replaced by $-\Gamma_{5}$. We analyse these projected equations by calculating the integrability conditions among them. The condition $\left[\partial_{z}, \partial_{r}\right] \epsilon=$ 0 requires $\partial_{z} f=0$ and yields a solution for $f$ given by

$$
f=2 g^{-1} \bar{a} \bar{\Lambda}+2 g^{-1} U\left(x^{+}\right) .
$$

The integrability $\left[\partial_{+}, \partial_{z}\right] \epsilon=0$ provides an equation for $U\left(x^{+}\right)$which is given by

$$
\mathrm{i} \frac{d U}{d x^{+}}-2 U^{2}-\frac{1}{4} c=0
$$

From the last line of eqs. (53) we have $f-2 g^{-1} \bar{a} \bar{\Lambda}=0$. This equation forces $U$ in the solution for $f$ to vanish. From the equation for $U$ we must in turn set $c=0$. Considering next the integrability condition $\left[\partial_{+}, \partial_{r}\right] \epsilon=0$ we first note that

$$
2 \Lambda f-\frac{1}{4} a g r H^{\prime}=\frac{c r^{2}-4 b}{8(g r)^{2}}\left(g_{1}-\mathrm{i} g_{2} \Gamma_{5}\right)
$$


It follows that the functions $S_{1}$ and $S_{2}$ must be constants. We need furthermore also to set $b=0$ (as well as imposing $g_{2}^{2} S_{1}=g_{1}^{2} S_{2}$ ). Letting $S_{i}=\mu_{i}$ the projected Killing spinor equations become

$$
\begin{aligned}
& {\left[\partial_{+}+\frac{\mathrm{i}}{\sqrt{2}}\left(\mu_{1}+\mu_{2}\right)\right] \epsilon=0, \quad \partial_{-} \epsilon=0, \quad \partial_{z} \epsilon=0,} \\
& {\left[\partial_{r}-\frac{\mathrm{i}}{2} \frac{g_{1}-\mathrm{i} g_{2} \Gamma_{5}}{\left(g_{1}^{2}+g_{2}^{2}\right)^{1 / 2}} f^{\prime} \Gamma_{-}-\frac{1}{2 r}\right] \epsilon=0 .}
\end{aligned}
$$

The Killing spinor solution is

$$
\epsilon=e^{-\frac{\mathrm{i}}{\sqrt{2}}\left(\mu_{1}+\mu_{2}\right) x^{+}} r^{1 / 2}\left[1+\frac{\mathrm{i}}{2} \frac{g_{1}-\mathrm{i} g_{2} \Gamma_{5}}{\left(g_{1}^{2}+g_{2}^{2}\right)^{1 / 2}} f \Gamma_{-}\right] \epsilon_{0},
$$

where $\epsilon_{0}$ is a constant spinor. Inserting the Killing spinor in the projection condition (152) and using

$$
f=\frac{\mu_{1}}{\sqrt{2} g_{1}^{2}\left(g_{1}^{2}+g_{2}^{2}\right)^{1 / 2} r^{2}}
$$

we obtain $\left(\Gamma_{r}+a\right) \epsilon_{0}=0$. Thus, the pp-wave preserves $\frac{1}{2}$ of the supersymmetry with $H$ given by

$$
H=-f^{2}=-\frac{\mu_{1}^{2}}{2 g_{1}^{4}\left(g_{1}^{2}+g_{2}^{2}\right) r^{4}}
$$

\section{$5 \quad$ PP-waves in six dimensions}

In this section we investigate the supersymmetry of pp-waves in Romans theory [30. We use the conventions of [31. We consider a subsector of the theory by truncating out the 2-form potential and the $U(1)$ potential. The Lagrangian describing the remaining fields is given by

$$
e^{-1} \mathcal{L}=R-\frac{1}{2}(\partial \varphi)^{2}-\frac{1}{4} X^{-2}\left(F_{(2)}^{a}\right)^{2}+4 g^{2}\left(X^{2}+\frac{4}{3} X^{-2}-\frac{1}{9} X^{-6}\right)
$$

where $X=e^{-\frac{1}{2 \sqrt{2}} \varphi}$ and $F_{(2)}^{a}=d A_{(1)}^{a}-\frac{1}{\sqrt{2}} g \epsilon_{a b c} A_{(1)}^{b} \wedge A_{(1)}^{c}$.

We have here set $g_{1}=g_{2}=-\sqrt{2} g$ in [31]. The supersymmetry transformations are

$$
\begin{aligned}
\delta \Psi_{M i} & =\left[D_{M}+\frac{1}{4} g\left(X+\frac{1}{3} X^{-3}\right) \Gamma_{M}\right] \epsilon_{i}-\frac{\mathrm{i}}{16 \sqrt{2}}\left(\Gamma_{M} \Gamma^{A B}-2 \Gamma^{A B} \Gamma_{M}\right) X^{-1} F_{A B i}{ }^{j} \epsilon_{j}, \\
\delta \lambda_{i} & =\left[-\frac{1}{2 \sqrt{2}} \Gamma^{M} \partial_{M} \varphi-\frac{1}{2} g\left(X-X^{-3}\right)\right] \epsilon_{i}-\frac{\mathrm{i}}{8 \sqrt{2}} \Gamma^{A B} X^{-1} F_{A B i}{ }^{j} \epsilon_{j},
\end{aligned}
$$

where $D_{M} \epsilon_{i}=\nabla_{M} \epsilon_{i}-\frac{\mathrm{i}}{\sqrt{2}} g A_{M i}{ }^{j} \epsilon_{j}$. We obtain the pp-wave with two of the $S U(2)$ fields turned off. The solution is given by

$$
\begin{aligned}
e^{2 A} & =(g r)^{4 / 3} H_{1}^{1 / 2}, \quad H_{1}=1+\frac{\ell_{1}^{2}}{r^{2}}, \\
e^{2 B} & =\frac{1}{(g r)^{2} H_{1}^{3 / 2}}, \quad e^{\sqrt{2} \varphi}=H_{1}, \\
A_{(1)}^{1} & =g^{-1} S_{1}\left(x^{+}\right)\left(1-H_{1}^{-1}\right) d x^{+},
\end{aligned}
$$


and the pp-wave function $H\left(x^{+}, r, z_{a}\right)$ satisfies the equation

$$
H^{\prime \prime}+\left(5 A^{\prime}-B^{\prime}\right) H^{\prime}+e^{-2(A-B)} \sum_{a} \partial_{a} \partial_{a} H+\frac{4 S_{1}^{2} \ell_{1}^{4}}{g^{2} r^{6}(g r)^{4 / 3} H_{1}^{4}}=0 .
$$

The Killing spinor equations are given by

$$
\begin{aligned}
& {\left[\partial_{+}+\frac{1}{2} A^{\prime} e^{A-B}\left(\Gamma_{+}+\frac{1}{2} H \Gamma_{-}\right)\left(\Gamma_{r}+1\right)-\frac{1}{4} e^{A-B} H^{\prime} \Gamma_{r} \Gamma_{-}-\frac{1}{4} \sum_{a} \partial_{a} H \Gamma_{a} \Gamma_{-}\right.} \\
& \left.\quad-\frac{\mathrm{i}}{\sqrt{2}} S_{1}\left(1-H_{1}^{-1}\right)\left(\Gamma_{r}+1\right)+\frac{\mathrm{i}}{4 \sqrt{2}} \frac{S_{1} \ell_{1}^{2}}{r^{2} H_{1}} \Gamma_{r} \Gamma_{+} \Gamma_{-}\right] \epsilon=0, \\
& {\left[\partial_{-}-A^{\prime} e^{A-B} \Gamma_{-}\left(\Gamma_{r}+1\right)\right] \epsilon=0,} \\
& {\left[\partial_{a}+\frac{1}{2} A^{\prime} e^{A-B} \Gamma_{a}\left(\Gamma_{r}+1\right)-\frac{\mathrm{i}}{4 \sqrt{2}} \frac{S_{1} \ell_{1}^{2}}{r^{2} H_{1}} \Gamma_{a} \Gamma_{r} \Gamma_{-}\right] \epsilon=0,} \\
& {\left[\partial_{r}+\frac{\ell_{1}^{2}+4 r^{2}}{12 r^{3} H_{1}} \Gamma_{r}+\frac{3 \mathrm{i}}{4 \sqrt{2}} \frac{S_{1} \ell_{1}^{2}(g r)^{1 / 3}}{g^{2} r^{4} H_{1}^{2}} \Gamma_{-}\right] \epsilon=0,} \\
& {\left[g\left(\Gamma_{r}+1\right)+\frac{\mathrm{i}}{\sqrt{2}} \frac{S_{1}}{(g r)^{2 / 3} H_{1}} \Gamma_{r} \Gamma_{-}\right] \epsilon=0 .}
\end{aligned}
$$

It is clear from these equations that the pp-waves preserve $\frac{1}{4}$ of the supersymmetry but there is no supernumerary supersymmetry.

\section{$6 \quad$ PP-waves in seven dimensions}

In this section we consider gauged $D=7, \mathcal{N}=2$ supergravity where we retain only the metric, two $U(1)$ gauge potentials and two scalars. The other fields are consistently set to zero. This reduced set of fields are described by the Lagrangian

$$
e^{-1} \mathcal{L}=R-\frac{1}{2}(\partial \vec{\varphi})^{2}-\frac{1}{4} \sum_{i=1}^{2} X_{i}^{-2}\left(F_{(2)}^{i}\right)^{2}-V
$$

where

$$
\begin{aligned}
& X_{i}=e^{-\frac{1}{2} \vec{a}_{i} \cdot \vec{\varphi}}, \quad \vec{a}_{1}=\left(\sqrt{2}, \sqrt{\frac{2}{5}}\right), \quad \vec{a}_{2}=\left(-\sqrt{2}, \sqrt{\frac{2}{5}}\right) \\
& V=\frac{1}{2} g^{2}\left(X_{1}^{-4} X_{2}^{-4}-8 X_{1} X_{2}-4 X_{1}^{-1} X_{2}^{-2}-4 X_{1}^{-2} X_{2}^{-1}\right) .
\end{aligned}
$$

The supersymmetry transformations are given by

$$
\begin{aligned}
\delta \psi_{M}= & {\left[\nabla_{M}+\frac{1}{4}\left(X_{1}^{-1} F_{M N}^{1} \Gamma_{12}+X_{2}^{-1} F_{M N}^{2} \Gamma_{34}\right) \Gamma^{N}+\frac{1}{4} g X_{1}^{-2} X_{2}^{-2} \Gamma_{M}\right.} \\
& \left.+\frac{1}{4}\left(X_{1}^{-1} \partial_{N} X_{1}+X_{2}^{-1} \partial_{N} X_{2}\right) \Gamma_{M} \Gamma^{N}+\frac{1}{2} g\left(A_{M}^{1} \Gamma_{12}+A_{M}^{2} \Gamma_{34}\right)\right] \epsilon, \\
\delta \lambda_{1}= & {\left[-\frac{1}{8}\left(3 X_{1}^{-1} \partial_{M} X_{1}+2 X_{2}^{-1} \partial_{M} X_{2}\right) \Gamma^{M}-\frac{1}{16} X_{1}^{-1} F_{A B}^{1} \Gamma^{A B} \Gamma_{12}+\frac{1}{4} g\left(X_{1}-X_{1}^{-2} X_{2}^{-2}\right)\right] \epsilon, } \\
\delta \lambda_{2}= & {\left[-\frac{1}{8}\left(2 X_{1}^{-1} \partial_{M} X_{1}+3 X_{2}^{-1} \partial_{M} X_{2}\right) \Gamma^{M}-\frac{1}{16} X_{2}^{-1} F_{A B}^{2} \Gamma^{A B} \Gamma_{34}+\frac{1}{4} g\left(X_{2}-X_{1}^{-2} X_{2}^{-2}\right)\right] \epsilon . }
\end{aligned}
$$


For more details see 32. The domain wall solution is given by

$$
\begin{aligned}
e^{2 A}=(g r)\left[H_{0}^{1 / 2} H_{1} H_{2}\right]^{\frac{1}{5}}, & H_{i}=1+\frac{\ell_{i}^{2}}{r^{2}}, \\
e^{2 B}=\frac{1}{(g r)^{2}\left[H_{0}^{1 / 2} H_{1} H_{2}\right]^{\frac{4}{5}}}, & X_{i}=H_{i}^{-1}\left[H_{0}^{1 / 2} H_{1} H_{2}\right]^{\frac{2}{5}},
\end{aligned}
$$

where $H_{0}=1+\ell_{0}^{2} / r^{2}$. The ansatz for the 1 -form potential is

$$
A_{(1)}^{i}=g^{-1} S_{i}\left(1-H_{i}^{-1}\right) d x^{+}
$$

and the function $H\left(x^{+}, r, z_{a}\right)$ satisfy the equation

$$
H^{\prime \prime}+\left(6 A^{\prime}-B^{\prime}\right) H^{\prime}+e^{-2(A-B)} \sum_{a} \partial_{a} \partial_{a} H+\frac{4 g^{2}}{r^{2}} e^{-10 A} \sum_{i} S_{i}^{2} \ell_{i}^{4} H_{i}^{-2}=0
$$

The Killing spinor equations are given by

$$
\begin{aligned}
& {\left[\partial_{+}+\frac{1}{4 r H_{0}} e^{A-B}\left(\Gamma_{+}+\frac{1}{2} H \Gamma_{-}\right)\left(\Gamma_{r}+1\right)-\frac{1}{4} e^{A-B} H^{\prime} \Gamma_{r} \Gamma_{-}-\frac{1}{4} \sum_{a} \partial_{a} H \Gamma_{a} \Gamma_{-}\right.} \\
& \left.\quad+\frac{1}{2}\left(S_{1}\left(1-H_{1}^{-1}\right) \Gamma_{12}+S_{2}\left(1-H_{2}^{-1}\right) \Gamma_{34}\right)\left(\Gamma_{r}+1\right)\right] \epsilon=0 \\
& {\left[\partial_{-}-\frac{1}{2 r H_{0}} e^{A-B} \Gamma_{-}\left(\Gamma_{r}+1\right)\right] \epsilon=0} \\
& {\left[\partial_{a}+\frac{1}{4 r H_{0}} e^{A-B} \Gamma_{a}\left(\Gamma_{r}+1\right)\right] \epsilon=0} \\
& {\left[\partial_{r}-\frac{1}{2 \sqrt{10}} \varphi_{2}^{\prime}+\frac{1}{4 r H_{0}} \Gamma_{r}-\frac{1}{2 r} g e^{-5 A}\left(S_{1} \ell_{1}^{2} H_{1}^{-1} \Gamma_{12}+S_{2} \ell_{2}^{2} H_{2}^{-1} \Gamma_{34}\right) \Gamma_{-}\right] \epsilon=0} \\
& {\left[g\left(\ell_{0}^{2}-\ell_{1}^{2}\right) H_{0}^{-1} X_{1}\left(\Gamma_{r}+1\right)+S_{1} \ell_{1}^{2} H_{1}^{-1} e^{-A} \Gamma_{12} \Gamma_{r} \Gamma_{-}\right] \epsilon=0} \\
& {\left[g\left(\ell_{0}^{2}-\ell_{2}^{2}\right) H_{0}^{-1} X_{2}\left(\Gamma_{r}+1\right)+S_{2} \ell_{2}^{2} H_{2}^{-1} e^{-A} \Gamma_{34} \Gamma_{r} \Gamma_{-}\right] \epsilon=0}
\end{aligned}
$$

It is clear from these equations that the pp-waves have $\frac{1}{4}$ supersymmetry but no supernumerary supersymmetry.

\section{Acknowledgments}

I am grateful to Hong Lü and Chris Pope for helpful discussions.

\section{References}

[1] R.R. Metsaev, Type IIB Green-Schwarz superstring in plane wave Ramond-Ramond background, Nucl. Phys. B625, 70 (2002), hep-th/0112044. 
[2] M. Blau, J. Figueroa-O'Farrill, C. Hull and G. Papadopoulos, "A new maximally supersymmetric background of IIB superstring theory, JHEP 0201, 047 (2002), hep-th/0110242.

[3] J. Kowalski-Glikman, Vacuum states in supersymmetric Kaluza-Klein theory, Phys. Lett. B134, 194 (1984).

[4] J.M. Maldacena, The large $N$ limit of superconformal field theories and supergravity, Adv. Theor. Math. Phys. 2231 (1998); Int. J. Theor. Phys. 381113 (1999), hep-th/9711200.

[5] S.S. Gubser, I.R. Klebanov and A.M. Polyakov, Gauge theory correlators from noncritical string theory, Phys. Lett. B428 105 (1998), hep-th/9802109.

[6] E. Witten, Anti-de Sitter space and holography, Adv. Theor. Math. Phys. 2253 (1998), hep-th/9802150.

[7] D. Berenstein, J.M. Maldacena and H. Nastase, Strings in flat space and pp-waves from $N=4$ super Yang Mills, JHEP 0204, 013 (2002), hep-th/0202021.

[8] M. Cvetič, H. Lü and C.N. Pope, Penrose limits, pp-waves and deformed M2-branes, Phys. Rev. D69, 046003 (2004), hep-th/0203082.

[9] M. Cvetič, H. Lü and C.N. Pope, M-theory pp-waves, Penrose limits and supernumerary supersymmetries, Nucl. Phys. B 644, 65 (2002), hep-th/0203229.

[10] J.P. Gauntlett and C.M. Hull, pp-waves in 11-dimensions with extra supersymmetry, JHEP 0206, 013 (2002), hep-th/0203255

[11] I. Bena and R. Roiban, Supergravity pp-wave solutions with 28 and 24 supercharges, Phys. Rev. D67, 125014 (2003), hep-th/0206195.

[12] J. Michelson, (Twisted) toroidal compactification of pp-waves, Phys. Rev. D66, 066002 (2002), hep-th/0203140.

[13] J. Michelson, A pp-wave with 26 supercharges, Class. Quant. Grav. 19, 5935 (2002), hep-th/0206204.

[14] V.R. Kaigorodov, Einstein spaces of maximum mobility, Dokl. Akad. Nauk. SSSR 146 793 (1962); Sov. Phys. Doklady 7, 893 (1963). 
[15] M. Cvetič, H. Lü and C.N. Pope, Spacetimes of boosted p-branes, and CFT in infinitemomentum frame, Nucl. Phys. B545, 309 (1999), hep-th/9810123.

[16] S.T.C. Siklos, Lobatchevski plane gravitational waves, in Galaxies, Axisymmetric systems and relativity, ed. M.A.H. MacCallum, Cambridge University Press, Cambridge (1985).

[17] I. Ozsvath, I. Robinson and K. Rozga, Plane-fronted gravitational and electromagnetic waves in spaces with cosmological constant, J. Math. Phys. 26 (1985) 1755.

[18] G.W. Gibbons and P.J. Ruback, Classical gravitons and their stability in higher dimensions, Phys. Lett. B171 (1986) 390.

[19] D. Brecher, A. Chamblin and H.S. Reall, AdS/CFT in the infinite momentum frame, Nucl. Phys. B607, 155 (2001), hep-th/0012076.

[20] J. Kerimo and H. Lü, PP-waves in AdS gauged supergravities and Supernumerary Supersymmetry, Phys. Rev. D71, 065003 (2005), hep-th/0408143.

[21] J.P. Gauntlett and J.B. Gutowski, All supersymmetric solutions of minimal gauged supergravity in five dimensions, Phys. Rev. D68, 105009 (2003), hep-th/0304064.

[22] M.M. Caldarelli and D. Klemm, All supersymmetric solutions of $N=2, D=4$ gauged supergravity, JHEP 0309, 019 (2003), hep-th/0307022

[23] S.L. Cacciatori, M.M. Caldarelli, D. Klemm and D.S. Mansi, More on BPS solutions of $N=2, D=4$ gauged supergravity, JHEP 0407, 061 (2004), hep-th/0406238

[24] R.G. Cai, Boosted domain wall and charged Kaigorodov space, Phys. Lett. B572, 75 (2003), hep-th/0306140.

[25] N.S. Deger and Ö. Sarioglu, Supersymmetric Strings and Waves in D=3, N=2 Matter Coupled gauged Supergravities, JHEP 0412 (2004) 039, hep-th/0409169.

[26] M. Cvetič, M.J. Duff, P. Hoxa, J.T. Liu, H. Lü, J.X. Lu, R. Martinez-Acosta, C.N. Pope, H. Sati and T.A. Tran, Embedding AdS black holes in ten and eleven dimensions Nucl. Phys. B558, 96 (1999), hep-th/9903214.

[27] M. Cvetič, S.S. Gubser, H. Lü and C.N. Pope, Symmetric potentials of gauged supergravities in diverse dimensions and Coulomb branch of gauge theories, Phys. Rev. D62 (2000) 086003, hep-th/9909121. 
[28] M.J. Duff and J.T. Liu, Anti-de Sitter black holes in gauged $\mathcal{N}=8$ supergravity, Nucl. Phys. B554 (1999) 237, hep-th/9901149.

[29] D.Z. Freedman and J.H. Schwarz, N=4 supergravity theory with local $S U(2) \times S U(2)$ invariance, Nucl. Phys. B137 (1978) 333.

[30] L.J. Romans, The F(4) gauged supergravity in six dimensions, Nucl. Phys. B269, 691 (1986).

[31] J.T. Liu, H. Lü and C.N. Pope, The radion mode in consistent brane-world reductions, hep-th/0212037.

[32] J.T. Liu and R. Minasian, Black holes and membranes in $A d S_{7}$, Phys. Lett. B457 39 , hep-th/9903269 